\title{
Moving Forward in Nursing Education through a Participatory Mode of Thinking / Aller de l'avant en formation infırmière en adoptant un mode de pensée participatif
}

Jacinthe I. Pepin

Université de Montréal, jacinthe.pepin@umontreal.ca

Florence Myrick

amyrick@ualberta.ca

Follow this and additional works at: https://qane-afi.casn.ca/journal

Part of the Nursing Commons, and the Scholarship of Teaching and Learning Commons

\section{Recommended Citation}

Pepin, Jacinthe I. and Myrick, Florence (2019) "Moving Forward in Nursing Education through a Participatory Mode of Thinking / Aller de l'avant en formation infirmière en adoptant un mode de pensée participatif," Quality Advancement in Nursing Education - Avancées en formation infirmière: Vol. 5: Iss. 2, Article 1.

DOI: https://doi.org/10.17483/2368-6669.1230

This Editorial is brought to you for free and open access by Quality Advancement in Nursing Education - Avancées en formation infirmière. It has been accepted for inclusion in Quality Advancement in Nursing Education - Avancées en formation infirmière by an authorized editor of Quality Advancement in Nursing Education - Avancées en formation infirmière. 


\section{Moving Forward in Nursing Education through a Participatory Mode of Thinking}

In nursing and other health sciences, many researchers adopt a participatory mode of thinking in approaching their studies not only with groups and communities of limited resources but also in various other contexts. Participatory research is defined by the Royal Society of Canada (RSC) as a "systematic investigation, with the collaboration of those affected by the issue being studied, for purposes of education and taking action or effecting social change" (https://www.mcgill.ca/familymed/research/projects/pram). Participatory studies or design projects in developing countries, in nursing and in engineering, for example, are viewed as a way of thinking collaboratively so that meaningful change in the communities, as much as knowledge creation, will be an end-result.

In nursing practice, Wolstenholme, Ross, Cobb, and Bowen (2017) provide such an example in an industrialized country, by describing the co-design of a service improvement project, where older people and other stakeholders were involved in the creative process. The authors found that the participatory or co-design practice inheres some of the underpinning philosophy of Person-Centred Nursing.

In nursing education, then, the question to be posed is could a participatory mode of thinking be espoused more readily to prepare nurses at the undergraduate and graduate levels to become effective collaborative change agents in the health care system? This approach would mean that nurses would learn to practice nursing from a participatory perspective in addition to learning to provide person or population-centred care. This process would equally mean that teachers in academic and clinical milieus would be called upon to collaborate more actively to create learning environments conducive to this participatory perspective together with a studentcentred perspective. Furthermore, a participatory mode of thinking would have to be adopted as an operational approach on care units, programs or directions, so that all nurses, including newly graduated nurses could participate in service improvement projects. Subsequently, nurses could become more efficient partners who, for example, would be more likely sought to participate in an Organizations' Boards of Directors for example.

Such an approach to nursing education specifically with regard to curricular development could actively involve both students and clinicians alike to be part of the process to design together with faculty members, programs, clinical activities, or courses, with a conscious effort to hear the voice of each group. It could generate the creation of fair assessment tools of the development of nursing competencies, such as clinical judgement or inter-professional collaboration, tools that are useful to both educational and practice milieus. In this issue of the Journal, Charette, Goudreau and Bourbonnais propose a model that integrates education and practice and Tchouaket et al. describe a tool developed collaboratively for the measurement of students' Professional Identity. Lapum et al. share their experience in developing an open educational e-textbook, which provides greater access to knowledge. Could we imagine going even further with a participatory mode of collaboration to advance quality in nursing education, and ultimately to bring forth meaningful change in the health care system?

Referring to the RSC's definition, "education and taking action or effecting social change" are essential ingredients of a participatory mode of thinking. Providing critical knowledge is one key for successful collaborative projects. Engaging the participants, students, educators and clinicians, is another aspect integral to designing change for specific care, professional, or system needs. Articles in this issue of the Journal address these essential 
components through their discussion of: Sustainable Development Goals (Sullivan, Bell, Haimour \& Richter), learning modalities that stimulate disorientating dilemma to facilitate transformation (Parzen \& Janzen), and learning activities that foster critical reflection and awareness of the social and professional worlds (Leclerc-Loiselle, Dufour, \& Pepin). In her interview on Governance education, Dr. Susan Duncan asked the following question: 'How do we prepare nurses for a governance process and equip them with the competencies and the lens of collaboration and transparency?'

A serious discussion is thus required regarding what we perceive as a paradox between our discourse on envisioning nurses as change agents and full participants in transforming the health system on the one hand and our limited opportunities to engage nursing students and nurses in the practice of participatory nursing, on the other hand.

\section{Aller de l'avant en formation infirmière en adoptant un mode de pensée participatif}

En sciences infirmières comme dans les autres sciences de la santé, plusieurs chercheurs abordent leurs projets de recherche en adoptant un mode de pensée participatif, non seulement avec les groupes et les communautés aux ressources limitées, mais dans divers autres contextes. La Société royale du Canada (SRC) décrit la recherche participative comme une « investigation systématique, menée en collaboration avec les personnes concernées par la question à l'étude, à des fins d'éducation et d'action ou de changement social. » (https://www.mcgill.ca/familymed/fr/recherche/projets/pram). Par exemple, dans les pays en voie de développement, les recherches participatives et les projets de design, en sciences infirmières et en ingénierie qui adoptent ce mode de pensée collaboratif visent un changement véritable au sein des communautés, tout en développant de nouvelles connaissances.

Dans la pratique infirmière, Wolstenholme, Ross, Cobb et Bowen (2017) fournissent un tel exemple pour un pays industrialisé, en décrivant la co-conception d'un projet d'amélioration des services, où des personnes âgées et d'autres parties prenantes étaient impliquées dans le processus créatif. Les auteurs ont constaté que la pratique participative ou la co-conception de projets est intrinsèquement liée aux fondements philosophiques des soins infirmiers axés sur la personne.

Ainsi, la question qui se pose pour la formation en sciences infirmières est la suivante : est-ce qu'un mode de pensée participatif pourrait être adopté plus assurément afin de préparer les infirmières, tant au premier cycle qu'aux cycles supérieurs, à devenir des agentes de changement plus efficaces au sein du système de santé? Une telle approche suppose que les infirmières apprendraient à exercer la pratique infirmière à partir d'une perspective participative tout en apprenant à offrir des soins centrés sur la personne ou la population. Ce processus signifierait également que les enseignantes, des milieux académiques et cliniques, seraient appelées à collaborer plus activement afin de créer des environnements d'apprentissage propices à cette perspective participative conjointement avec la perspective centrée sur les étudiantes. Par ailleurs, un mode de pensée participatif aurait avantage à être adopté en tant qu'approche opérationnelle dans les unités, programmes ou directions des soins, afin que l'ensemble des infirmières, incluant les infirmières nouvellement diplômées, puissent participer aux projets d'amélioration des soins et services. Par conséquent, celles-ci pourraient devenir des partenaires plus efficaces, et il est probable qu'elles pourraient être appelées, par exemple, à participer au sein de conseils d'administration de diverses organisations. 
Un tel mode de pensée en formation infirmière, s'observe notamment lors de l'élaboration de programmes, par l'implication active d'étudiantes, de cliniciennes et de membres de la faculté, au processus de conception de programmes, d'activités cliniques ou de cours, en faisant consciemment l'effort d'être à l'écoute de chaque groupe. Ce mode pourrait mener à la création d'outils conjoints pour une évaluation juste du développement des compétences infirmières, telles que le jugement clinique ou la collaboration interprofessionnelle; des outils qui seraient utiles autant aux milieux d'enseignement que de pratique. Charette, Goudreau et Bourbonnais, dans ce numéro, proposent un modèle qui intègre les milieux d'enseignement en sciences infirmières et les milieux cliniques, alors que Tchouaket et coll. décrivent un outil conçu en collaboration afin de mesurer l'identité professionnelle des étudiantes. Lapum et coll. partagent leur expérience de l'élaboration d'un livre électronique de formation en libre accès, offrant ainsi un plus grand accès aux connaissances. Pouvons-nous imaginer aller encore plus loin dans le mode participatif de collaboration pour promouvoir la qualité de la formation en sciences infirmières et ultimement, pour produire des changements importants au sein du système de santé?

Se référant à la définition de la SRC, «à des fins d'éducation et d'action ou de changement social » sont des éléments essentiels au mode de pensée participatif. Fournir des connaissances d'importance critique est un facteur clé dans la réussite de projets collaboratifs. Impliquer les participants, étudiants, enseignants et cliniciens, est un autre aspect indissociable à l'élaboration de stratégies de changements pour répondre à des besoins de soins spécifiques, à des besoins professionnels ou à ceux du système. Des articles dans ce numéro du journal abordent ces éléments essentiels en discutant : des objectifs de développement durable (ODD) (Sullivan, Bell, Haimour et Richter), des modes d'apprentissage qui stimulent les dilemmes déconcertants afin de faciliter la transformation des apprenantes (Parzen et Janzen) et d'activités d'apprentissage qui favorisent la réflexion critique et une consciences des mondes sociaux et professionnels (Leclerc-Loiselle, Dufour et Pepin). Dans le cadre de son entrevue sur la formation à la gouvernance, la $\mathrm{D}^{\text {re }}$ Susan Duncan pose la question suivante : "Comment préparons-nous les infirmières au processus de gouvernance et comment pouvons-nous les soutenir dans le développement des compétences requises dans une optique de transparence et de collaboration?»

À cet égard, une importante discussion est nécessaire en en ce qui a trait à ce que nous percevons comme un paradoxe entre, d'une part, le discours sur notre vision des infirmières comme agentes du changement et participantes à part entière au processus de transformation du système de santé et, d'autre part, les possibilités limitées que nous fournissons aux étudiantes infirmières et aux infirmières de s'engager dans la pratique participative des soins infirmiers.

Jacinthe Pepin, RN PhD, Co-Editor-in-Chief - Corédactrice en chef

Florence Myrick, RN PhD, Co-Editor-in-Chief - Corédactrice en chef

\section{References / Références}

Royal Society Canada. (2019). Participatory Research at McGill (PRAM). Retrieved from https://www.mcgill.ca/familymed/research/projects/pram 
Société royale du Canada. (2019). Recherche participative à l'Université McGill. Réparé à https://www.mcgill.ca/familymed/fr/recherche/projets/pram

Wolstenholme, D., Ross, H., Cobb, M., \& Bowen, S. (2017). Participatory design facilitates Person Centred Nursing in service improvement with older people: a secondary directed content analysis. Journal of clinical nursing, 26(9-10), 1217-1225. https://doi.org/10.1111/jocn.13385 